\title{
El estado de la digitalización de América Latina frente a la pandemia de la COVID-19
}

Revista Latinoamericana de Economía y Sociedad Digital

Issue 1, agosto 2020

Autores: Raúl Katz(D), Fernando Callorda(iD), Juan Jung(iD)

DOI: $10.53857 / G Q O L 2178$

Publicado: 10 agosto, 2020

Cita sugerida: Katz, Raúl, Fernando Callorda \& Juan Jung (2020) “El estado de la digitalización de América Latina frente a la pandemia de la COVID-19" en Revista Latinoamericana de Economía y Sociedad Digital, Issue 1

Licencia: Creative Commons Atribución-NoComercial 4.0 Internacional (CC BY-NC 4.0)

Tipo: Ensayo

Palabras clave: $\underline{\text { COVID-19, Economía Digital, Telecomunicaciones }}$

\section{Resumen}

El propósito de este artículo es evaluar el posicionamiento de la digitalización en América Latina para enfrentar a la COVID-19. En este sentido, nuestro objetivo es evaluar la resiliencia del ecosistema digital en múltiples dimensiones. Comenzamos recogiendo evidencia de la afectación de redes y enrutadores Wi-Fi ante el aumento de tráfico. A continuación, desarrollamos una serie de indicadores para evaluar la resiliencia digital de los hogares, del aparato productivo, del mercado laboral y del Estado para hacer frente a la pandemia. Concluimos identificando recomendaciones para mejorar el desempeño del ecosistema digital en contextos como el actual. Al respecto, entendemos conveniente facilitar los procedimientos para despliegues de infraestructuras móviles, asignar espectro adicional de forma temporal, incentivar a proveedores de streaming a reducciones de tráfico, aumentar porciones de espectro no licenciado para enrutadores Wi-Fi, promover plataformas que superen falencias en cadenas de aprovisionamiento, estimular el teletrabajo y fortalecer la capacitación de sectores vulnerables. 


\section{Introducción}

La pandemia de la COVID-19 es inusitada en la medida en que plantea un desafío al sistema socioeconómico mundial. El fenómeno afecta por igual a economías avanzadas y a países emergentes dado que, tanto por sus efectos como por las políticas públicas puestas en práctica, representa un replanteamiento de prácticas sociales y de sistemas productivos que hasta finales del año pasado se daban por normales. Numerosos analistas anticipan una recesión global este año debido a la crisis causada por el coronavirus. Por ejemplo, en su reciente World Economic Outlook, el Fondo Monetario Internacional proyecta que la economía mundial sufrirá una brusca contracción de 4,9\% en 2020, peor que la registrada durante la crisis financiera de 2008-09. Para América Latina y el Caribe, el citado organismo anticipa una caída de 9,4\% este año.

A partir de la aplicación de las primeras medidas sanitarias para responder a la pandemia, sumadas al temor por el contagio, comenzaron a acumularse las evidencias anecdóticas que daban cuenta de la importancia de las tecnologías digitales para contrarrestar el aislamiento, difundir medidas profilácticas y facilitar el funcionamiento de sistemas económicos. Entre los indicadores más claros, figuran el incremento exponencial del tráfico de Internet (y el consecuente desafío para los operadores de preservar niveles de calidad adecuados), la importancia del teletrabajo y la necesidad de mantener activas las cadenas de aprovisionamiento y de distribución de bienes. En este contexto, analizaremos el nivel de preparación de los diferentes países de América Latina para hacer frente a las disrupciones planteadas. Para ello, evaluaremos la resiliencia del ecosistema digital ${ }^{[1]}$ frente a la pandemia de la COVID-19 en múltiples dimensiones: infraestructura, hogares, sistemas productivos, estructura laboral y el Estado.

Para el abordaje de los contenidos planteados, el presente artículo se estructura de la siguiente forma. El capítulo 2 sintetiza el estado del ecosistema digital en la región. El capítulo 3 da cuenta de los niveles de resiliencia de las infraestructuras. El capítulo 4 analiza la resiliencia a escala de los hogares. El capítulo 5 se focaliza en el análisis del aparato productivo. El capítulo 6 mide la resiliencia a nivel de los Estados. Por último, el capítulo 7 finaliza con las conclusiones principales.

\section{El estado del ecosistema digital en América Latina}

Nuestra investigación presentada en otros trabajos (Katz et al. 2020b) indica que la digitalización contribuye a mitigar parcialmente los efectos socioeconómicos de una pandemia. Por ejemplo, el análisis econométrico realizado para medir el rol de la digitalización frente a los efectos del SARS en 2003 indica que aproximadamente el $75 \%$ de las pérdidas económicas asociadas con esa pandemia han podido ser mitigadas en aquellos países con buena infraestructura de conectividad. De manera similar, la magnitud de la reducción en las proyecciones de crecimiento económico presentadas por el Fondo 
Monetario Internacional está inversamente correlacionada con el avance en la digitalización de la producción (Katz 2020). Si la digitalización es un factor importante para mitigar el efecto de pandemias, es importante establecer cuál es su desarrollo en América Latina.

América Latina y el Caribe están posicionados en un nivel de desarrollo intermedio respecto a otras regiones del mundo en términos de desarrollo de su ecosistema digital (Katz et al. 2020a). Con un índice de 49,92 ${ }^{[2]}$ (en una escala de 0 a 100), la región está en una posición más avanzada respecto a África $(35,05)$ y a Asia Pacífico $(49,16)$. Sin embargo, a pesar de los avances significativos de los últimos 15 años en el desarrollo de su ecosistema digital, América Latina y el Caribe todavía muestran un rezago respecto a Europa Occidental (con un índice de 71,06), América del Norte $(80,85)$, Europa del Este $(52,90)$ y los Estados Árabes agrupados en torno al acrónimo MENA $(55,54)$. A este rezago se suma una tasa de crecimiento anual del índice inferior a la de otras regiones. En efecto, América Latina y el Caribe pertenecen al grupo de países emergentes que presentan una moderada tasa de crecimiento anual de su digitalización (ver Gráfico 1).

Gráfico 1. Índice CAF de Desarrollo del Ecosistema Digital (2018) vs. Tasa de crecimiento (2004-18)

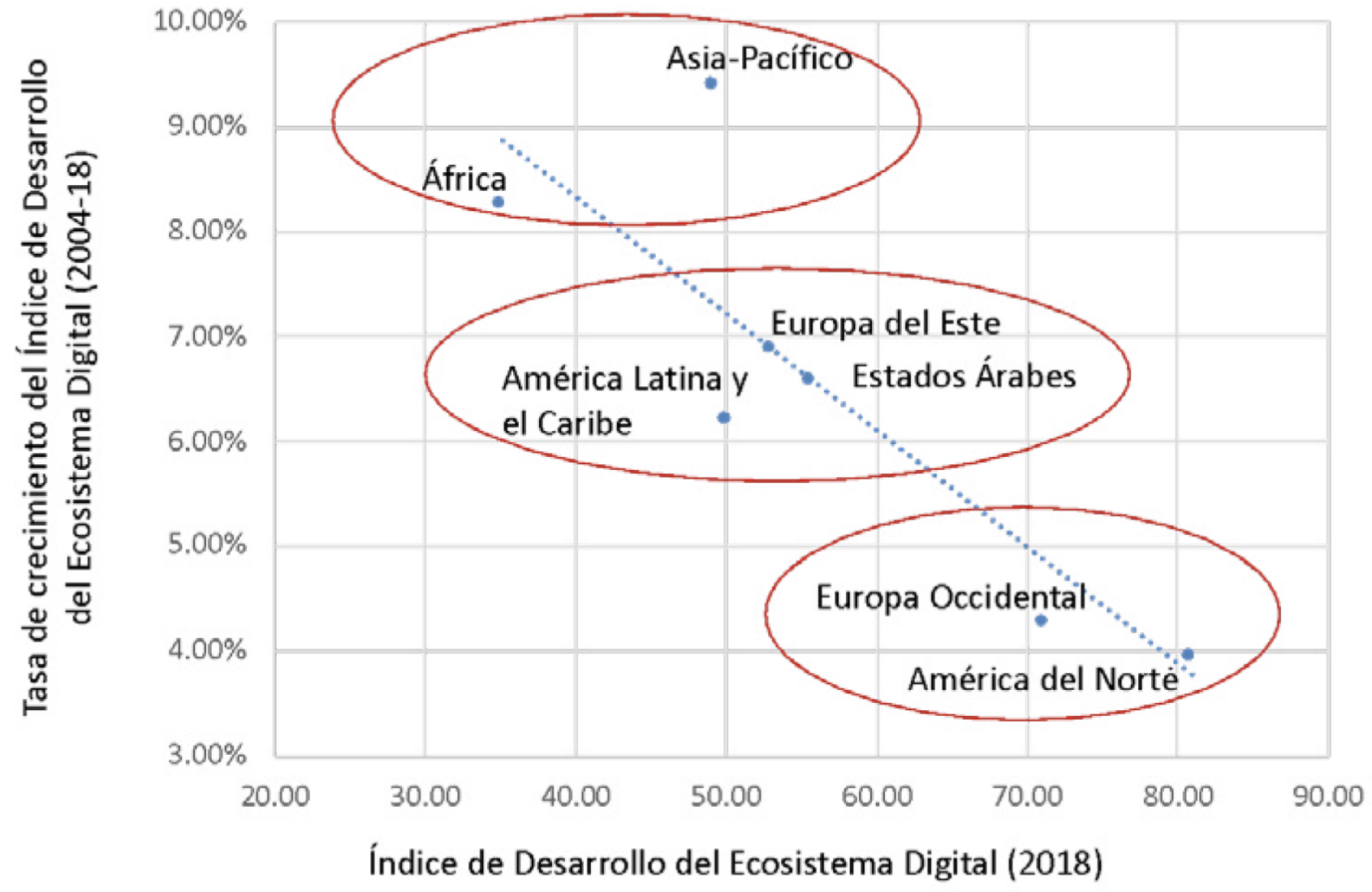

Fuente: Análisis Telecom Advisory Services. 
Como se observa en el Gráfico 1, el índice de desarrollo del ecosistema digital de América Latina y el Caribe presenta una tasa anual de crecimiento compuesto entre el $2004 \mathrm{y} \mathrm{el}$ 2018 de 6,21 \%, la más baja entre los países emergentes. De hecho, de acuerdo con su nivel de desarrollo del ecosistema digital, América Latina y el Caribe deberían presentar una tasa de crecimiento más acelerada. La desagregación del índice por los pilares que lo componen permite identificar los grandes desafíos que enfrenta la región para lidiar con la disrupción de la pandemia (ver Gráfico 2).

Gráfico 2. Índice CAF de Desarrollo del Ecosistema Digital (2018)
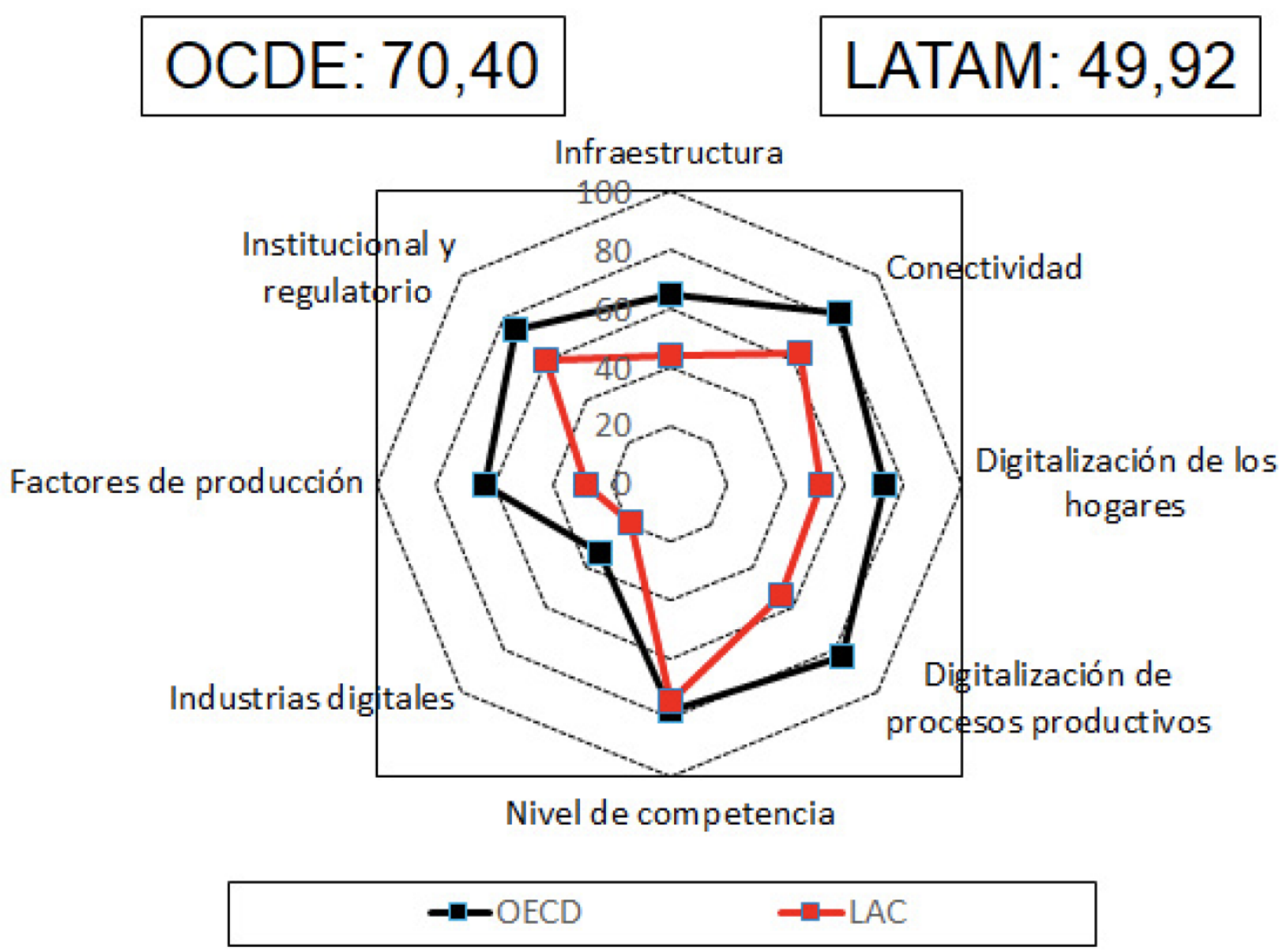

Fuente: Katz et al. (2020a).

En los siguientes capítulos evaluaremos la situación actual de cuatro pilares de la digitalización: la infraestructura de redes, la digitalización de los hogares, la digitalización de la producción y la digitalización del Estado. 


\section{Resiliencia de la infraestructura digital}

La imposición de medidas sanitarias para enfrentar la COVID-19, como la clausura de lugares de trabajo y la cuarentena domiciliaria, ha ocasionado un salto en el uso de las redes de telecomunicaciones ${ }^{[3]}$ para resolver temas de aprovisionamiento de bienes, conectividad social y acceso a información; como consecuencia se generó una erosión natural de los índices de calidad. Por ejemplo, de acuerdo con Ookla/Speedtest, los índices de velocidad promedio y de latencia en Internet en la región sufrieron cambios importantes en las últimas semanas de marzo (Cuadro 1).

Cuadro 1. Desempeño de redes de Internet frente a la COVID-19 ${ }^{[4]}$ 


\begin{tabular}{|c|c|c|c|c|c|}
\hline Indicador & Período & Brasil & Chile & Ecuador & México \\
\hline \multirow{6}{*}{ Velocidad promedio banda ancha fija (Mbps) } & Febrero 2020 & 52,57 & 92,96 & 27,35 & 36,55 \\
\hline & Semana del 9 de marzo & 54,42 & 104,29 & 24,85 & 38,08 \\
\hline & Semana del 16 de marzo & 52,89 & 90,87 & 22,34 & 37,14 \\
\hline & Semana del 23 de marzo & 51,88 & 91,25 & 23,95 & 36,80 \\
\hline & Semana del 30 de marzo & 52,30 & 98,65 & 23,47 & 36,06 \\
\hline & Semana del 6 de abril & 52,50 & 98,72 & 22,61 & 35,74 \\
\hline \multirow{5}{*}{ Latencia banda ancha fija (ms) } & Febrero 2020 & 17 & 38 & 23 & 41 \\
\hline & Marzo 2020 & 18 & 39 & 29 & 43 \\
\hline & Semana del 9 de marzo & 17 & 21 & 17 & 27 \\
\hline & Semana del 16 de marzo & 19 & 24 & 19 & 28 \\
\hline & Semana del 30 de marzo & 19 & 25 & 19 & 29 \\
\hline \multirow{6}{*}{ Velocidad promedio banda ancha móvil (Mbps) } & Febrero 2020 & 24,20 & 19,51 & 20,65 & 26,99 \\
\hline & Semana del 9 de marzo & 25,04 & 19,94 & 19,94 & 26,62 \\
\hline & Semana del 16 de marzo & 24,30 & 16,23 & 20,51 & 26,74 \\
\hline & Semana del 23 de marzo & 23,72 & 15,56 & 19,45 & 28,26 \\
\hline & Semana del 30 de marzo & 25,11 & 16,22 & 20,77 & 28,60 \\
\hline & Semana del 6 de abril & 25,23 & 16,30 & 20,77 & 29,68 \\
\hline \multirow{5}{*}{ Latencia banda ancha móvil (ms) } & Febrero 2020 & 46 & 41 & 40 & 50 \\
\hline & Marzo 2020 & 47 & 43 & 39 & 48 \\
\hline & Semana del 9 de marzo & 48 & 40 & 38 & 50 \\
\hline & Semana del 16 de marzo & 48 & 46 & 38 & 51 \\
\hline & Semana del 30 marzo & 49 & 48 & 40 & 50 \\
\hline
\end{tabular}

Fuente: Ookla/Speedtest.

De acuerdo con los valores del Cuadro 1, la velocidad y la latencia de banda ancha en marzo demuestra alta volatilidad, probablemente reflejando picos en el acceso a Internet, combinados con cambios en el comportamiento y en el flujo de tráfico (del lugar de trabajo o estudio a la casa). El análisis desde febrero hasta la última semana de marzo muestra lo siguiente:

- Velocidad de banda ancha fija: reducción gradual por semana en Brasil y en México, con una marcada disminución en Chile en marzo; disminución constante en Ecuador desde febrero hasta la primera semana de abril.

- Latencia de banda ancha fija: gradual incremento (y por lo tanto, erosión de calidad de servicio) en Brasil (11,7 \%), en Chile (19,0 \%), en Ecuador (11,8 \%) y en México $(7,4 \%)$.

- Velocidad de banda ancha móvil: estable en Brasil y en México; disminución en Chile (notable) y en Ecuador (gradual). Estos últimos fueron los países que decretaron una cuarentena nacional. 
- Latencia de banda ancha móvil: estable en todos los países, aunque a niveles altos.

Existe numerosa evidencia empírica acerca del impacto que la velocidad de banda ancha ejerce en el crecimiento económico (Rohman y Bohlin 2012, Kongaut y Bohlin 2014, Briglauer y Gugler 2018, Carew et al. 2018, Ford 2018, Grimes et al. 2009, Mack-Smith 2006). Recientemente, Katz y Callorda (2019) analizaron el impacto de la velocidad de banda ancha fija en el PIB de 159 países entre 2008 y 2019, y demostraron el mencionado efecto positivo (ver Cuadro 2).

Cuadro 2. Impacto de la velocidad de descarga de la banda ancha fija en el PIB

\begin{tabular}{|c|c|c|c|}
\hline Impacto en el PIB & $\begin{array}{l}\text { Velocidad } \\
<10 \text { Mbps }\end{array}$ & $\begin{array}{l}\text { Velocidad } \\
10 \text { Mbps - } 40 \\
\text { Mbps }\end{array}$ & $\begin{array}{l}\text { Velocidad }>\mathbf{4 0} \\
\text { Mbps }\end{array}$ \\
\hline $\begin{array}{l}\text { Ln Velocidad de } \\
\text { descarga- } 4\end{array}$ & $\begin{array}{l}-0,00206 \\
(0,00136)\end{array}$ & $\begin{array}{l}0,00264 \\
(0,00138) * * *\end{array}$ & $\begin{array}{l}0,00730 \\
(0,00211) * * *\end{array}$ \\
\hline Ln Empleo tt & $\begin{array}{l}0,00664 \\
(0,00189) * * *\end{array}$ & $\begin{array}{l}0,00525 \\
(0,00168) * * *\end{array}$ & $\begin{array}{l}0,00458 \\
(0,00165) * * *\end{array}$ \\
\hline Ln Inversión tt- 4 & $\begin{array}{l}0,01459 \\
(0,00216) * * *\end{array}$ & $\begin{array}{l}-0,00616 \\
(0,00382)\end{array}$ & $\begin{array}{l}-0,00085 \\
(0,00481)\end{array}$ \\
\hline Número de países & 116 & 105 & 49 \\
\hline Observaciones & 2.113 & 1.792 & 575 \\
\hline R-Square & 0,9516 & 0,9262 & 0,9438 \\
\hline
\end{tabular}

Nota: Todas las estimaciones incluyen efectos fijos por país, por trimestre, y controles por crecimiento del PIB del período anterior y por penetración de banda ancha. Errores estándar robustos entre paréntesis. ${ }^{*} \mathrm{p}<10 \%, * * \mathrm{p}<5 \%, * * * \mathrm{p}<1 \%$.

Fuente: Análisis Telecom Advisory Services.

Como lo indican los resultados del Cuadro 2, el coeficiente de impacto económico de la velocidad de banda ancha fija aumenta con la velocidad: de no significativo a velocidades inferiores a $10 \mathrm{Mbps}$, hasta 0,73025 si la velocidad de descarga se incrementa $100 \%$ para 
países con velocidad de descarga igual o superior a $40 \mathrm{Mbps}$. La evidencia inversa es válida, es decir, la disminución de la velocidad de descarga tendrá un efecto económico inverso. Si bien la disminución de velocidad de descarga como resultado de la pandemia se limita a marzo, corresponde hacer la pregunta de si esta situación se perpetúa, el impacto económico negativo podría materializarse.

En este contexto, cabe indagar si es posible limitar los efectos negativos del aumento del tráfico en la calidad de las redes. Naturalmente, las medidas que incluyan el despliegue adicional de infraestructura para acomodar el pico en el tráfico requieren un lapso relativamente prolongado. Sin embargo, tres iniciativas pueden ser tomadas con impacto relativamente corto. La primera es el despliegue de mayor cantidad de radiobases para banda ancha móvil. Este esfuerzo es una iniciativa que todo operador toma normalmente para enfrentar saltos circunstanciales en tráfico. Para acelerar el proceso, sería conveniente flexibilizar los permisos necesarios para permitir a los operadores reaccionar rápidamente. La segunda iniciativa es asignar a operadores móviles espectro adicional de manera temporaria (por ejemplo, como hizo recientemente la FCC en Estados Unidos) ${ }^{[5]}$. La tercera es requerir a proveedores de servicios de video streaming la reducción en sus volúmenes de tráfico ${ }^{[6]}$. Ciertos operadores ya han respondido: Google anunció la reducción en la calidad de definición de YouTube; Disney demoró el lanzamiento de su servicio de Disney+ en Francia y Microsoft limitó el ritmo de actualización de sus plataformas de juegos en Xbox.

Uno de los efectos más claros de la pandemia ha sido la clausura de lugares de trabajo y de enseñanza para prevenir el contagio, y el aumento drástico del teletrabajo. Más allá del impacto en las redes de telecomunicaciones, el teletrabajo ha generado impactos en aplicaciones de videoconferencia (para facilitar la comunicación laboral) y el tráfico de datos dentro del hogar basado en la tecnología Wi-Fi. Por ejemplo, el tráfico de Webex, la plataforma de Cisco, al conectarse a China aumentó 22 veces desde el comienzo de la pandemia, mientras que el número de usuarios en Japón, Corea del Sur y Singapur se incrementó entre 4 y 5 veces ${ }^{[7]}$.

El aumento natural en el número de dispositivos conectados en el hogar que utilizan plataformas de videoconferencia y trabajo en la nube ha creado un cuello de botella en los enrutadores Wi-Fi que operan sobre espectro no-licenciado (ver Gráfico 2).

Como puede observarse en el Gráfico 2, desde fines de marzo las estadísticas de tráfico provenientes de 125 millones de enrutadores Wi-Fi alrededor del mundo indican un aumento del $80 \%$ en las cargas de PC a la nube, así como picos ocasionados por el uso de videoconferencias. Esto crea una saturación en bandas de espectro no licenciado (generalmente en $2.4 \mathrm{GHz}$ y $5 \mathrm{GHz}$ ). Los reguladores deberán comenzar a examinar la necesidad de aumentar la porción de espectro no licenciado en bandas superiores de $5 \mathrm{GHz}$ y $6 \mathrm{GHz}$ (siguiendo el ejemplo de Estados Unidos).

Gráfico 3. Global: Aumento en tráfico de Wi-Fi (Diciembre 2019-Abril 2020) 


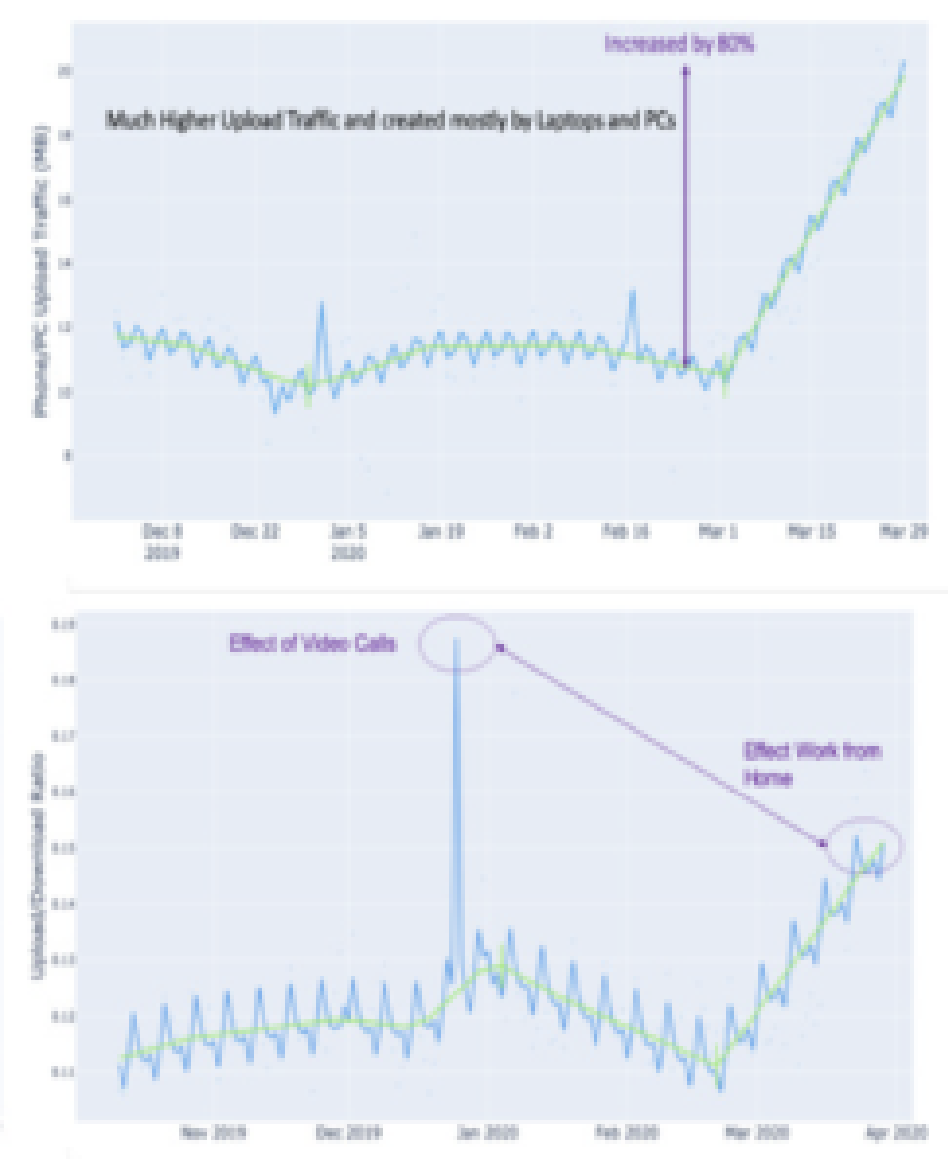

Fuente: Assia (2020).

En conclusión, la infraestructura digital es un componente fundamental para la resiliencia económica. La capacidad de las redes para acomodar las necesidades de comunicación resultantes de COVID-19 requiere de la acción conjunta de operadores, reguladores y plataformas de Internet.

\section{Resiliencia de los hogares digitales}

La digitalización de hogares permite a la población continuar realizando una cantidad de tareas cotidianas que anteriormente requerían el contacto físico. De acuerdo con las últimas estadísticas disponibles, el porcentaje de penetración de Internet en América Latina es 68,66 \% . Extrapolando la tasa de penetración por país al 2020, se llega a una penetración ponderada promedio de 78,78 \%. Este valor revela, por un lado, el avance de la tecnología para poder afrontar el confinamiento impuesto por la pandemia, aunque también demuestra un obstáculo para afrontar la COVID-19 mediante el uso de tecnologías digitales. La marginalización implícita del 21 \% de la población en el uso de Internet excluye una porción importante de los habitantes de la posibilidad de acceder a servicios que pueden reemplazar algunas actividades que requieren habitualmente el contacto físico. Como es de esperar, 
esta marginalización varía por país (ver Cuadro 3).

Cuadro 3. Penetración de Internet y uso de redes sociales 


\begin{tabular}{|c|c|c|c|c|c|c|}
\hline \multirow{2}{*}{ País } & \multicolumn{3}{|c|}{ Penetración de Internet } & \multicolumn{3}{|c|}{ Penetración de Facebook } \\
\hline & 2018 & 2019 & 2020 & 2018 & 2019 & 2020 \\
\hline Argentina & $77,78 \%$ & $81,42 \%$ & $85,24 \%$ & $68,07 \%$ & $71,26 \%$ & $74,60 \%$ \\
\hline Barbados & $84,03 \%$ & $86,37 \%$ & $88,77 \%$ & $55,94 \%$ & $57,50 \%$ & $59,10 \%$ \\
\hline Bolivia & $48,22 \%$ & $53,04 \%$ & $58,34 \%$ & $53,59 \%$ & $58,95 \%$ & $64,85 \%$ \\
\hline Brasil & $74,22 \%$ & $81,64 \%$ & $89,80 \%$ & $66,67 \%$ & $68,00 \%$ & $69,36 \%$ \\
\hline Chile & $82,33 \%$ & $82,33 \%$ & $82,33 \%$ & $69,33 \%$ & $69,33 \%$ & $69,33 \%$ \\
\hline Colombia & $66,68 \%$ & $71,40 \%$ & $76,47 \%$ & $58.19 \%$ & $62,32 \%$ & $66,74 \%$ \\
\hline Costa Rica & $74,09 \%$ & $76,88 \%$ & $79,79 \%$ & $63,72 \%$ & $66,13 \%$ & $68,62 \%$ \\
\hline Ecuador & $60,67 \%$ & $64,27 \%$ & $68,09 \%$ & $58,74 \%$ & $62,23 \%$ & $65,93 \%$ \\
\hline El Salvador & $37,20 \%$ & $40,92 \%$ & $45,02 \%$ & $51,18 \%$ & $56,30 \%$ & $61,93 \%$ \\
\hline Guatemala & $71,50 \%$ & $78,65 \%$ & $86,52 \%$ & $39,39 \%$ & $43,33 \%$ & $47,66 \%$ \\
\hline Honduras & $34,06 \%$ & $36,60 \%$ & $39,33 \%$ & $36,05 \%$ & $38,74 \%$ & $41,62 \%$ \\
\hline Jamaica & $60,58 \%$ & $66,64 \%$ & $73,30 \%$ & $38,46 \%$ & $42,31 \%$ & $46,54 \%$ \\
\hline México & $65,77 \%$ & $67,75 \%$ & $69,79 \%$ & $62,53 \%$ & $64,41 \%$ & $66,35 \%$ \\
\hline Panamá & $62,01 \%$ & $66,45 \%$ & $71,20 \%$ & $48,09 \%$ & $51,53 \%$ & $55,22 \%$ \\
\hline Paraguay & $64,99 \%$ & $69,16 \%$ & $73,60 \%$ & $46,79 \%$ & $49,79 \%$ & $52,98 \%$ \\
\hline Perú & $52,54 \%$ & $56,65 \%$ & $61,08 \%$ & $62,19 \%$ & $67,05 \%$ & $72,30 \%$ \\
\hline República Dominicana & $74,82 \%$ & $82,31 \%$ & $90,54 \%$ & $49,68 \%$ & $54,65 \%$ & $60,11 \%$ \\
\hline Trinidad y Tobago & $81,58 \%$ & $86,06 \%$ & $90,79 \%$ & $50,91 \%$ & $53,71 \%$ & $56,66 \%$ \\
\hline Uruguay & $70,21 \%$ & $72,20 \%$ & $74,24 \%$ & $68,45 \%$ & $70,39 \%$ & $72,38 \%$ \\
\hline Venezuela & $79,20 \%$ & $87,12 \%$ & $95,83 \%$ & $45,05 \%$ & $49,55 \%$ & $54,50 \%$ \\
\hline América Latina & $68,66 \%$ & $73,52 \%$ & $78,78 \%$ & $61,29 \%$ & $63,83 \%$ & $66,51 \%$ \\
\hline OCDE & $83,93 \%$ & $86,07 \%$ & $88,33 \%$ & $62,78 \%$ & $64,48 \%$ & $66,28 \%$ \\
\hline
\end{tabular}


Nota: Datos del 2019 y el 2020 han sido extrapolados sobre la base de la tasa de crecimiento reciente.

Fuente: Unión Internacional de Telecomunicaciones; Internet World Stats; análisis Telecom Advisory Services.

El promedio ponderado para la región indica el progreso de los últimos años en términos de adopción de Internet. Sin embargo, los promedios nacionales esconden diferencias significativas en el interior de cada país. A modo ilustrativo, la adopción de Internet suele mostrar niveles muy superiores en áreas urbanas en relación con las rurales (por ejemplo, es el caso de Bolivia, Brasil o Ecuador). Otro aspecto para considerar es el número de dispositivos de acceso en el hogar. Si bien la tenencia de computadoras en América Latina alcanza $44,89 \%{ }^{[8]}$, en gran parte de los hogares esto no sería suficiente para acomodar el acceso simultáneo de varios miembros de la familia.

Más allá de la adopción de Internet, el análisis de su utilización revela un comportamiento de uso que reduce su contribución a la resiliencia del hogar para enfrentar la pandemia. En general, la banda ancha es usada como medio de comunicación y para redes sociales ${ }^{[9]}$ (ver, por ejemplo, penetración de Facebook en el Cuadro 5). Asimismo, un factor que reduce el poder de resiliencia de la digitalización del hogar para afrontar la pandemia es el hecho de que aun entre los usuarios de Internet la capacidad para adoptar servicios que permitan "virtualizar" actividades físicas es limitada. Para medir la capacidad de hogares digitales para encarar actividades por medio de Internet, hemos creado un "índice de resiliencia digital del hogar" que combina cuatro indicadores:

- Número de apps relacionadas con el cuidado de la salud bajadas por año por habitante: asumimos que si un usuario baja aplicaciones de salud es más propenso a utilizar Internet para obtener información sobre comportamiento sanitario, evolución de la pandemia, puntos de realización de test, etc. (fuente: App Annie ${ }^{[10]}$ ).

- Número de apps educativas bajadas por año por habitante: asumimos que la descarga de aplicaciones educativas brinda una indicación de la capacidad para continuar proveyendo educación a niños en el hogar (fuente: App Annie).

- Número de plataformas fintech por millón de habitantes: se asume que la densidad de plataformas fintech de un país está naturalmente alineada con una demanda para realizar transacciones monetarias (fuente: Crunchbase).

- Porcentaje del comercio electrónico de todo el comercio minorista: indicador del uso de comercio electrónico para realizar operaciones de compra de comida y artículos de consumo en el hogar (fuente: Euromonitor).

Cuanto más altos sean los indicadores de adopción de cada uno de estos cuatro 
componentes, mejor estará preparada la población para afrontar condiciones de aislamiento y cuarentena (Cuadro 4).

Cuadro 4. Indicadores de uso de plataformas digitales (2020)

\begin{tabular}{|c|c|c|c|c|}
\hline País & $\begin{array}{l}\text { Uso de } \\
\text { Internet para } \\
\text { apps de } \\
\text { salud (1) }\end{array}$ & $\begin{array}{l}\text { Uso de Internet } \\
\text { para apps } \\
\text { educativas (2) }\end{array}$ & $\begin{array}{l}\text { Densidad de } \\
\text { plataformas } \\
\text { fintech (3) }\end{array}$ & $\begin{array}{l}\text { Intensidad de } \\
\text { comercio } \\
\text { electrónico (4) }\end{array}$ \\
\hline Argentina & 9,27 & 52,62 & 0,87 & $6,73 \%$ \\
\hline Bolivia & 3,41 & 7,11 & 1,33 & $0,94 \%$ \\
\hline Brasil & 10,59 & 65,22 & 1,07 & $7,84 \%$ \\
\hline Chile & 12,08 & 87,35 & 2,98 & $3,70 \%$ \\
\hline Colombia & 6,79 & 50,73 & 2,36 & $6,15 \%$ \\
\hline Ecuador & 3,97 & 9,09 & 1,14 & $3,29 \%$ \\
\hline El Salvador & 5,50 & 13,34 & 0,57 & $3,03 \%$ \\
\hline Guatemala & 1,99 & 7,13 & 0,18 & $2,86 \%$ \\
\hline Honduras & 3,56 & 10,83 & 0,57 & $2,42 \%$ \\
\hline México & 4,44 & 48,19 & 0,57 & $4,57 \%$ \\
\hline Panamá & 10,41 & 24,75 & 0,91 & $8,03 \%$ \\
\hline Paraguay & 5,94 & 12,59 & 1,22 & $4,97 \%$ \\
\hline Perú & 5,56 & 52,35 & 2,46 & $1,86 \%$ \\
\hline $\begin{array}{l}\text { República } \\
\text { Dominicana }\end{array}$ & 5,58 & 16,89 & 0,95 & $4,21 \%$ \\
\hline América Latina & 7,59 & 51,02 & 1,16 & $5,70 \%$ \\
\hline OCDE & 15,19 & 76,07 & 5,05 & $11,52 \%$ \\
\hline
\end{tabular}


Nota: Ciertos países fueron excluidos de esta lista por falta de confiabilidad en los datos fuente.

Fuentes: (1) y (2) App Annie, (3) Crunchbase, (4) Euromonitor; análisis Telecom Advisory Services.

Al combinar estos cuatro indicadores en un índice de "resiliencia digital del hogar", se puede visualizar cuáles son los países cuya población está más preparada para afrontar la cuarentena sanitaria mediante la digitalización de hogares (ver Cuadro 5).

Cuadro 5. Índice de resiliencia digital del hogar 


\begin{tabular}{|c|c|}
\hline País & Índice \\
\hline Argentina & 33,87 \\
\hline Bolivia & 6,23 \\
\hline Brasil & 40,59 \\
\hline Chile & 41,14 \\
\hline Colombia & 31,69 \\
\hline Ecuador & 11,53 \\
\hline El Salvador & 12,78 \\
\hline Guatemala & 8,70 \\
\hline Honduras & 9,83 \\
\hline México & 25,63 \\
\hline Panamá & 28,63 \\
\hline Paraguay & 16,90 \\
\hline Perú & 23,33 \\
\hline República Dominicana & 16,38 \\
\hline América Latina & 30,70 \\
\hline OCDE & 53,78 \\
\hline
\end{tabular}


Fuente: Análisis Telecom Advisory Services.

La primera observación a partir de los índices del Cuadro 5 es la diferencia que existe entre América Latina y los países de la OCDE, lo que indica que los hogares de estos últimos están mejor preparados digitalmente para afrontar la pandemia. La segunda conclusión es la heterogeneidad del índice dentro de la región. Argentina, Brasil, Chile y Colombia muestran un nivel más alto de preparación que el resto de las naciones. Esto significa que, de manera agregada, la posibilidad de hogares para acceder a información sanitaria, realizar transacciones monetarias, adquirir bienes por comercio electrónico y contribuir a la educación de niños en países con un índice inferior a 30 es más limitada. Como es de esperar, si se pudiese desagregar el índice por grupo social, se podría ver cómo esto afecta principalmente a las poblaciones más vulnerables.

\section{Resiliencia de la producción}

La digitalización de la producción representa el factor fundamental para mantener la economía operando en situaciones como la actual. Para analizar el grado de "resiliencia productiva", consideramos dos dimensiones: (i) la digitalización de procesos productivos y (ii) el grado de digitalización del trabajo. En el primer caso, evaluamos hasta qué punto el sistema productivo puede seguir operando bajo las condiciones actuales, y nos referimos especialmente a las cadenas de aprovisionamiento, el procesamiento y los canales de distribución. En el segundo caso, se analiza el grado de preparación de una economía para afrontar la migración al teletrabajo.

\subsection{Digitalización de la cadena de aprovisionamiento}

En este análisis, definimos la cadena de aprovisionamiento de manera más extendida que la mera adquisición de insumos por parte de firmas (Calatayud y Katz 2019). Por tanto, consideramos la multiplicidad de actores que ocupan funciones específicas a lo largo de la cadena de suministros, con la participación no solo de proveedores de insumos, empresas manufactureras y canales de comercialización, sino también de los actores que facilitan el flujo de productos y de información a lo largo de la cadena. Para su funcionamiento armónico, estas cadenas requieren tanto prestadores de servicios logísticos, financieros y de tecnología como instituciones públicas que faciliten el desarrollo de la infraestructura y la construcción de un clima de negocios adecuado (ver Figura 1).

Figura 1. Principales actores de una cadena logística 


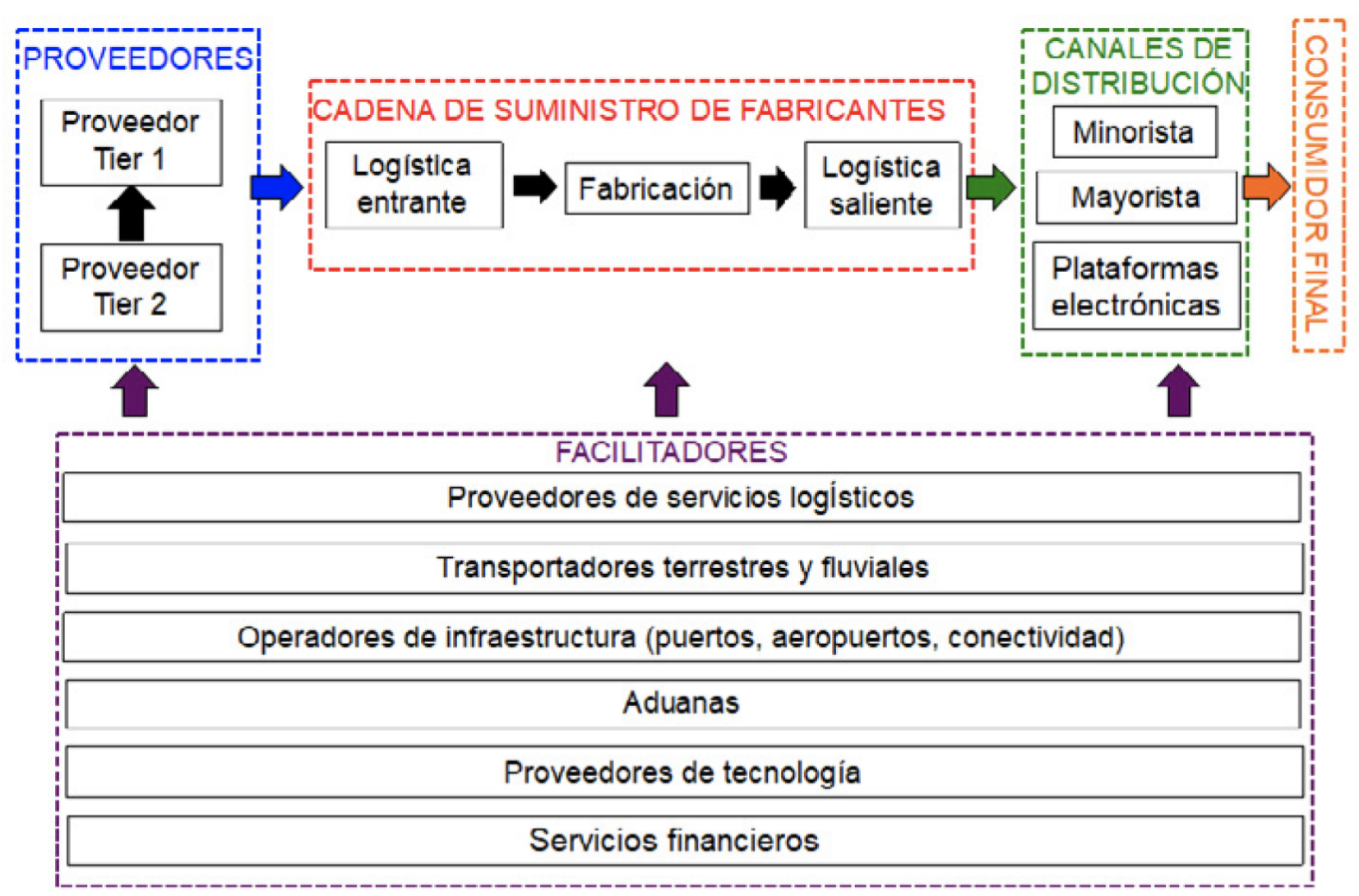

Fuente: Calatayud y Katz (2019).

De esta forma, los principales actores que participan en una cadena logística incluyen:

- Proveedores de insumos de primer y segundo nivel. Los de primer nivel son quienes abastecen de insumos directamente a las grandes empresas manufactureras. Los de segundo nivel son quienes abastecen de insumos a las empresas proveedoras de primer nivel, y se constituyen así en proveedores indirectos de las grandes empresas manufactureras.

- Empresas manufactureras. Normalmente, son empresas de gran tamaño y pertenecientes a diferentes industrias como, por ejemplo, automotriz, alimentación o textil. Para llegar a un producto final, estas empresas utilizan diferentes insumos en su proceso de transformación, los cuales son suplidos por proveedores de primer nivel.

- Mayoristas y minoristas. Se trata de empresas en el sector de la comercialización, que canalizan los productos de empresas manufactureras hacia los consumidores finales.

- Proveedores de servicios logísticos, empresas de transporte y operadores de infraestructura. Esto incluye las empresas que facilitan el movimiento físico de insumos y productos finales, y que brindan servicios de almacenamiento, embalaje y gestión de 
inventario.

- Organismos de control. Se refiere a instituciones públicas que intervienen en el proceso, especialmente en caso de importación y exportación, a fin de asegurar que cumplan con las normativas arancelarias, de seguridad, sanitarias y fitosanitarias.

- Proveedores de tecnología. Incluye empresas que proveen sistemas y tecnologías para la gestión digital de procesos, su automatización.

- Proveedores de servicios financieros. Incluye bancos y entidades financieras que facilitan el acceso a financiamiento de inversión y de capital circulante para empresas de la cadena de suministro, mediante instrumentos tales como préstamos, factoring, garantías y leasing.

La implicancia de este marco analítico es que, para que un sistema productivo funcione con cierta resiliencia en el marco de la pandemia, todos los actores que participan en una cadena de aprovisionamiento deben presentar un grado avanzado de digitalización. No alcanza con que las empresas manufactureras estén suficientemente digitalizadas para manejar órdenes de compra de insumos de manera digital, sino que deben estar preparadas para interactuar con las empresas logísticas para tener suficiente información sobre la trazabilidad de las mercancías en tránsito, o estar preparadas para interactuar con los operadores portuarios y las aduanas para hacer eficiente el transporte de productos.

Si bien las empresas latinoamericanas presentan una alta adopción a Internet, la proporción de estas que usan la plataforma en su cadena de aprovisionamiento es más reducida (ver Cuadro 6).

Cuadro 6. Digitalización de la cadena de aprovisionamiento (2018)

\begin{tabular}{|l||l|l||l|}
\hline País & $\begin{array}{l}\text { Empresas conectadas a } \\
\text { Internet }\end{array}$ & $\begin{array}{l}\text { Empresas que } \\
\text { usan banca electrónica }\end{array}$ & $\begin{array}{l}\text { Empresas que usan Internet para } \\
\text { adquirir insumos }\end{array}$ \\
\hline \hline Argentina & $94,30 \%$ & $79,60 \%$ & $45,80 \%$ \\
\hline \hline Brasil & $96,40 \%$ & $88,00 \%$ & $66,00 \%$ \\
\hline \hline Chile & $86,16 \%$ & $84,37 \%$ & $28,80 \%$ \\
\hline \hline Colombia & $92,81 \%$ & $95,39 \%$ & $37,00 \%$ \\
\hline \hline Ecuador & $93,89 \%$ & $47,06 \%$ & $13,90 \%$ \\
\hline \hline México & $94,61 \%$ & $76,60 \%$ & $13,47 \%$ \\
\hline \hline Perú & $94,00 \%$ & $34,20 \%$ & $15,20 \%$ \\
\hline \hline Uruguay & $93,39 \%$ & $68,35 \%$ & $38,43 \%$ \\
\hline
\end{tabular}


Nota: La diferencia entre países se debe principalmente al grado de inclusión de pymes en la muestra.

Fuentes: INDEC. Encuesta Nacional sobre Innovación y Conducta Tecnológica (2004-2011)resultados extrapolados; Centro Regional de Estudos para o Desenvolvimento da Sociedade da Informação. Pesquisa sobre o uso das TIC nas empresas brasileiras - TIC empresas (2017); Ministerio de Economía, Fomento y Turismo. División de Política Comercial e Industrial. Encuesta Longitudinal de Empresas (2017); Ministerio TIC y Cámara de Comercio de Bogotá. Observatorio de la Economía Digital de Colombia (2017); Ecuador. Instituto Nacional de Estadística y Censos. Empresas y TIC: Módulo de TIC de las Encuestas de Manufactura y Minería, Comercio Interno y servicios (2015); Perú: Instituto Nacional de Estadística e Informática. Encuesta Económica Anual; Tecnología de Información y Comunicación en las empresas; análisis Telecom Advisory Services.

Las estadísticas del Cuadro 6 indican que, más allá del acceso a Internet, una porción importante de empresas (principalmente pymes) no ha incorporado la tecnología en su cadena de aprovisionamiento. Además de lo presentado en el Cuadro 6, existe una importante variación entre sectores industriales y entre países. Por ejemplo, de manera general, el sector automotriz presenta un grado de preparación más avanzado que el resto de los sectores. Aun así, dentro del sector existen diferencias entre países. Al analizar la situación del sector automotriz por país, se observa que México es el país más avanzado en el diseño de estrategias tecnológicas y la adopción de nuevas tecnologías (en parte debido a la integración vertical con empresas de Estados Unidos), seguido por Brasil.

Aun para aquellas empresas que hayan digitalizado internamente su cadena de aprovisionamiento, existen barreras para la comunicación interorganizacional entre todos los participantes de la cadena de suministros. En la última década, la mayor parte de países de la región han mejorado el desempeño de algunos de los facilitadores de la cadena de suministro como, por ejemplo, la infraestructura de puertos, carreteras interurbanas y aeropuertos. Esto se ve reflejado en una mejor posición de la región en los rankings internacionales de logística. Pese a ello, la región se encuentra aún muy lejos de los niveles de desempeño de las economías avanzadas. Por ejemplo, el bajo nivel de digitalización de la industria de transporte terrestre representa un importante cuello de botella para la eficientización de la cadena de suministro. La industria latinoamericana de transporte terrestre está excesivamente fragmentada, compuesta por una mayoría de empresas pequeñas, con baja capacidad de inversión, limitaciones en capacidad de implementación tecnológica y acceso limitado a recursos financieros. Como ejemplo, de las aproximadamente 150.000 empresas de transporte terrestre en México, solamente 10 tienen suficiente escala para llevar adelante una transformación digital ${ }^{[11]}$. En Colombia, de los 3500 proveedores de transporte terrestre ${ }^{[12]}$, se estima que tan solo 100 pueden llegar a adoptar tecnologías digitales ${ }^{[13]}$. En este contexto, empresas tecnológicas con el perfil de 
plataforma de vinculación digital (matching platforms) están siendo desarrolladas para proporcionar una relación más eficiente entre proveedores logísticos y servicios de transporte ${ }^{[14]}$. Estas firmas suministran servicios digitales con el objeto de resolver los fallos de coordinación entre los actores involucrados.

Con relación a la gestión de los procesos aduaneros, en los últimos años se registraron ciertos avances en la simplificación y digitalización de estos, incluyendo el desarrollo en muchos países de la ventanilla única de comercio exterior. Aun así, los países de América Latina todavía están rezagados respecto a las mejores prácticas internacionales. Esto se evidencia, por ejemplo, en el tiempo requerido para procesar documentación necesaria para comercio exterior (ver Cuadro 7).

Cuadro 7. Horas requeridas para procesar documentación de comercio exterior (2018)

\begin{tabular}{|c|c|c|}
\hline País & Exportación & Importación \\
\hline Argentina & 30 & 192 \\
\hline Brasil & 12 & 48 \\
\hline Colombia & 60 & 64 \\
\hline México & 8 & 18 \\
\hline Paraguay & 24 & 36 \\
\hline
\end{tabular}

Benchmarks extrarregionales

\begin{tabular}{|l||l||l|}
\hline \hline Países Bajos & 1 & 1 \\
\hline \hline Singapur & 2 & 3 \\
\hline
\end{tabular}

Fuente: Banco Mundial. Doing Business 2018.

En resumen, la digitalización limitada de las cadenas de aprovisionamiento en el interior de empresas, principalmente pymes, y los cuellos de botella en las cadenas logísticas representan obstáculos para el desarrollo de cadenas resilientes para hacer frente a la 
COVID-19.

\subsection{Digitalización de canales de distribución}

Las barreras a la digitalización en la cadena de aprovisionamiento en la región se extienden a los canales de distribución. El Cuadro 8 presenta una compilación de estadísticas sobre el porcentaje de empresas que han desplegado canales de venta digitales o que han desarrollado sitios web.

Cuadro 8. Digitalización de canales de distribución (2018)

\begin{tabular}{|c||l||l|}
\hline País & $\begin{array}{l}\text { Porcentaje de } \\
\text { empresas que tienen sitio } \\
\text { web }\end{array}$ & $\begin{array}{l}\text { Porcentaje de empresas que han } \\
\text { desplegado canales de venta } \\
\text { digitales }\end{array}$ \\
\hline \hline Argentina & $63,60 \%$ & $18,52 \%$ \\
\hline \hline Brasil & $59,52 \%$ & $22,00 \%$ \\
\hline \hline Chile & $78,80 \%$ & $10,60 \%$ \\
\hline \hline Colombia & $67,21 \%$ & $38,00 \%$ \\
\hline \hline Ecuador & --- & $9,20 \%$ \\
\hline \hline México & $49,79 \%$ & $8,68 \%$ \\
\hline \hline Perú & --- & $7,20 \%$ \\
\hline \hline Uruguay & $52,75 \%$ & $35,41 \%$ \\
\hline
\end{tabular}

Nota: La información para algunos países se estimó basándose en la correlación con el nivel de adopción de Internet.

Fuente: UNCTAD; análisis Telecom Advisory Services.

Como puede observarse, si bien numerosas empresas han desarrollado sitios web para aumentar su visibilidad, muchas menos tienen la capacidad de recibir órdenes de compra 
electrónicas. La manera más rápida de resolver esta barrera es apalancar y acelerar el desarrollo de plataformas de comercio electrónico que permitan introducir un nivel de eficiencia en la distribución de bienes.

\subsection{La transformación de la fuerza de trabajo}

Los efectos sobre el tráfico en las redes de la irrupción masiva del teletrabajo ya han sido abordados en la sección 3. Otro abordaje refiere a sus consecuencias en el mercado laboral y sus implicancias sociales. Para ello, procuraremos indagar acerca de cuántos trabajadores han migrado a sus hogares, y establecer perspectivas respecto de cuáles son los sectores más y menos afectados. En principio, se podría considerar que los trabajadores del conocimiento (por ejemplo, investigadores, y desarrolladores de software) serían más propensos a adoptar los nuevos modos de trabajo. Una pregunta relevante es: ¿cuál es el número de trabajadores que, por la naturaleza de su ocupación, no pueden trabajar desde el hogar? Tan solo en marzo, 6.650.000 desocupados solicitaron cobrar el seguro de desempleo en Estados Unidos. ¿Cómo podría ser esta situación en América Latina?

Para ello recurrimos a la Encuesta de Caracterización Socioeconómica Nacional (CASEN) del Ministerio de Desarrollo Social de Chile realizada en 2017. La encuesta dispone de información para más de 200.000 individuos, que representan (usando los códigos de expansión) toda la población del país. En la medida en que cada encuestado responde en primer lugar sobre si está empleado o no, las observaciones consideradas incluyen solamente a aquellos individuos que están en la fuerza laboral y ocupados en el momento de la encuesta. El objetivo es aplicar para cada una de las 387 ocupaciones la probabilidad de que deban seguir operando en el lugar físico (por ejemplo, los profesionales de la salud deben continuar trabajando en hospitales), así como la probabilidad de migración al teletrabajo (esta última basada en el tipo de ocupación). Las probabilidades fueron determinadas basándose en decretos oficiales que indicaban qué trabajadores debían concurrir al lugar de trabajo y quiénes no, complementado con nuestro análisis subjetivo (ver ejemplo en Cuadro 9).

Cuadro 9. Ejemplo de probabilidades de teletrabajo 


\begin{tabular}{|c|c|c|c|}
\hline CIUO-88 & Empleo & $\begin{array}{l}\text { Probabilidad } \\
\text { Teletrabajo }\end{array}$ & $\begin{array}{l}\text { Servicio en } \\
\text { cuarentena }\end{array}$ \\
\hline 3442 & Funcionarios del fisco & $100,00 \%$ & $0,00 \%$ \\
\hline 3443 & $\begin{array}{l}\text { Funcionarios de servicios de } \\
\text { seguridad social }\end{array}$ & $100,00 \%$ & $0,00 \%$ \\
\hline 3444 & $\begin{array}{l}\text { Funcionarios de servicios de } \\
\text { expedición de licencias y } \\
\text { permisos }\end{array}$ & $50,00 \%$ & $0,00 \%$ \\
\hline 3449 & $\begin{array}{l}\text { Agentes de las } \\
\text { administraciones públicas de } \\
\text { aduanas, impuestos y afines, } \\
\text { no clasificados bajo otros } \\
\text { epígrafes }\end{array}$ & $50,00 \%$ & $0,00 \%$ \\
\hline
\end{tabular}

Fuente: CASEN 2017; análisis Telecom Advisory Services.

La compilación de probabilidades indica el porcentaje de la fuerza laboral que puede seguir trabajando en el hogar, el porcentaje que debe seguir concurriendo a su lugar de trabajo y el porcentaje que no puede recurrir al teletrabajo ni está obligado a seguir trabajando (ver figura 2).

Figura 2. Chile: Análisis de impacto en el teletrabajo 


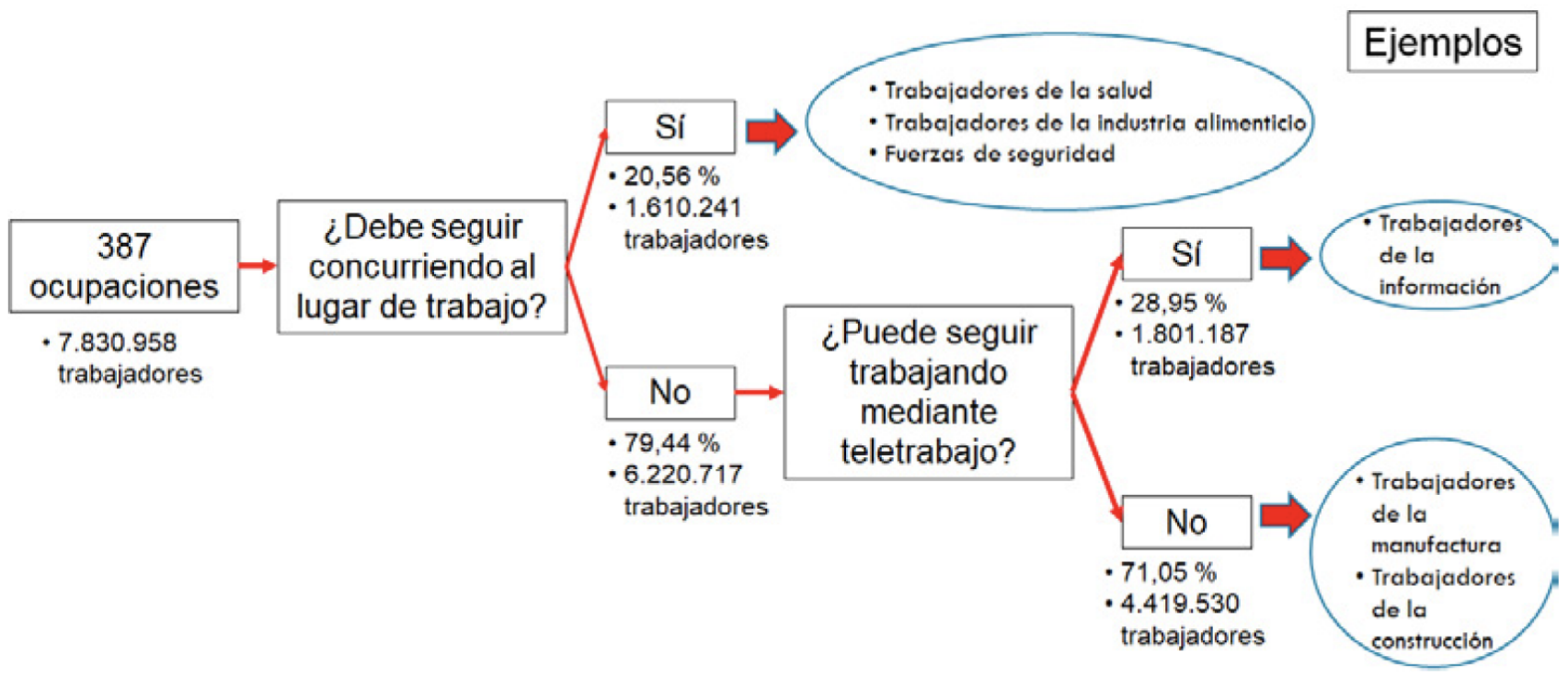

Fuente: Encuesta CASEN; análisis Telecom Advisory Services.

El análisis muestra los siguientes resultados:

- Del total de la fuerza laboral censada en 2017 (excluyendo desocupados, pero incluyendo empleo informal), Chile contaba con 7.830 .958 trabajadores.

- Del total de 7.830.958, 1.610.241 (o sea 20,56 \%) deben continuar yendo a su lugar de trabajo (por ejemplo, personal de sanidad, fuerzas de seguridad, etc.).

- Del total nuevamente, 6.220 .717 (79,44 \%) no pueden concurrir a su lugar de trabajo.

- Del universo de 6.220.717 que no pueden concurrir a su lugar de trabajo, 1.801.187 (o sea $28,95 \%$ ) pueden cumplir sus funciones mediante teletrabajo en el hogar. Este es el número que determina el salto en la utilización de redes analizado en la sección 3.

- Finalmente, del universo de 6.220.717, 4.419.530 (o sea 71,05 \%) no pueden trabajar remotamente desde el hogar.

- Del universo de 7.830.958, los trabajadores que no concurren al lugar de trabajo y que no pueden cumplir sus funciones mediante teletrabajo representan el 56,44 \%.

Estos valores son de una magnitud significativa en lo que respecta a su implicancia social. De esta fuerza laboral, una proporción perderá el empleo dado que la empresa en la que trabaja cesará en sus operaciones, mientras que otros podrán continuar en plantilla, aunque las empresas podrán determinar (si lo permite la legislación laboral) si se continúa pagando un sueldo o no. Las implicancias sociales son aún más graves cuando se analiza a los trabajadores en situación de vulnerabilidad, que no pueden concurrir al lugar de trabajo y 
tampoco teletrabajar (Cuadro 10).

Cuadro 10. Chile: Discriminación de impacto de COVID-19 en el empleo

\begin{tabular}{|c|c|c|c|c|}
\hline $\begin{array}{l}\text { Estadísticas } \\
\text { Chile } 2017\end{array}$ & $\begin{array}{l}\text { Porcentaje } \\
\text { que } \\
\text { mantiene el } \\
\text { empleo }\end{array}$ & $\begin{array}{l}\text { Tienen que } \\
\text { seguir } \\
\text { asistiendo } \\
\text { al trabajo }\end{array}$ & $\begin{array}{l}\text { Trabajan a } \\
\text { distancia }\end{array}$ & $\begin{array}{l}\text { No pueden } \\
\text { trabajar }\end{array}$ \\
\hline $\begin{array}{l}\text { Toda la } \\
\text { población }\end{array}$ & $43,56 \%$ & 1.610 .241 & 1.801 .187 & 4.419 .530 \\
\hline $\begin{array}{l}\text { Sin educación } \\
\text { básica }\end{array}$ & $27,87 \%$ & 169.614 & 29.828 & 516.194 \\
\hline $\begin{array}{l}\text { Educación } \\
\text { básica }\end{array}$ & $30,55 \%$ & 384.599 & 98.872 & 1.098 .905 \\
\hline Educación media & $37,81 \%$ & 676.925 & 429.245 & 1.819 .559 \\
\hline $\begin{array}{l}\text { Educación } \\
\text { superior }\end{array}$ & $62,63 \%$ & 369.701 & 1.234 .063 & 957.042 \\
\hline $\begin{array}{l}\text { 1er quintil de } \\
\text { ingreso }\end{array}$ & $29,59 \%$ & 192.495 & 61.732 & 604.999 \\
\hline $\begin{array}{l}\text { 2do quintil de } \\
\text { ingreso }\end{array}$ & $33,34 \%$ & 342.862 & 149.758 & 985.042 \\
\hline $\begin{array}{l}\text { 3er quintil de } \\
\text { ingreso }\end{array}$ & $37,91 \%$ & 392.459 & 267.170 & 1.080 .271 \\
\hline $\begin{array}{l}\text { 4to quintil de } \\
\text { ingreso }\end{array}$ & $44,42 \%$ & 407.535 & 436.918 & 1.056 .494 \\
\hline $\begin{array}{l}\text { 5to quintil de } \\
\text { ingreso }\end{array}$ & $63,27 \%$ & 274.586 & 885.610 & 673.520 \\
\hline
\end{tabular}


Fuente: CASEN 2017; análisis Telecom Advisory Services.

- De toda la fuerza de trabajo de Chile 7.830.958, 43,56 \% conserva el empleo porque o tienen que continuar asistiendo al lugar de trabajo $(20,56 \%)$ o pueden trabajar remotamente (23\%). Lógicamente, por la dificultad de mantener el distanciamiento social, el 20,56 \% que continúa asistiendo al lugar de trabajo tiene más riesgo de contagio.

- Ello implica que más de 56 \% de la fuerza de trabajo enfrenta condiciones de disrupción. Este porcentaje es similar al calculado por Hevia y Neumeyer (2020), que indican que un 53 $\%$ de la fuerza laboral está en riesgo en América Latina y el Caribe ya que trabajan en empresas de hasta 5 empleados con limitado acceso a instituciones financieras.

- De los 1.801.187 trabajadores que pueden trabajar remotamente, 1.234.063 tienen educación superior, y 1.322.528 están en el 4to y 5to quintil de ingreso.

- De los 4.419.530 que no pueden asistir al trabajo y no pueden trabajar remotamente, 1.615,099 tienen, como máximo, un nivel de educación básica; del mismo universo, 1.590.041 pertenecen al 1er o 2do quintil de ingreso.

Más allá de las implicancias sociales inmediatas, es fundamental trabajar sobre la reestructuración de procesos del sector productivo para incrementar el porcentaje de la población que pueda trabajar remotamente, así como enfatizar la capacitación de los sectores sociales más vulnerables.

\section{Resiliencia del Estado}

La resiliencia del Estado frente a la pandemia está basada en su capacidad para mantener en funcionamiento sus procesos administrativos, así como para continuar entregando servicios públicos. Naturalmente, existen servicios no prescindibles cuya continuidad está menos condicionada por el nivel de digitalización (por ejemplo, la seguridad y la salud pública). Por otra parte, la digitalización de otros servicios puede aumentar su capacidad de afrontar la COVID-19.

Un ejemplo en este sentido es el de la digitalización en los trámites vinculados al procesamiento de documentación requerida para el comercio exterior. Resulta evidente que, a mayor digitalización de estos procesos, mayores serán las posibilidades de asegurar su continuidad en situaciones de cierre o de limitaciones horarias de oficinas públicas. Bajo el supuesto de que la agilidad en torno a estos trámites depende de la posibilidad de realizarlos de forma online, es posible utilizar el indicador de los tiempos requeridos para este tipo de gestiones como proxy del nivel de digitalización a nivel del Estado, sin desmedro de que puedan existir otros ejemplos igualmente válidos. Como se mencionó antes, pese a los avances que han registrado los países de la región en este indicador, aún queda mucho por hacer para alcanzar los niveles de los países más avanzados (como se ilustró en el Cuadro 7). 
Otra métrica que puede dar una perspectiva de la digitalización de servicios públicos es el índice del desarrollo de gobierno electrónico (Cuadro 11).

Cuadro 11. Índice de desarrollo de gobierno electrónico

\begin{tabular}{|c|c|}
\hline País & Valor del índice \\
\hline Argentina & 0,73 \\
\hline Bolivia & 0,53 \\
\hline Brasil & 0,73 \\
\hline Chile & 0,74 \\
\hline Colombia & 0,69 \\
\hline Ecuador & 0,61 \\
\hline El Salvador & 0,55 \\
\hline Guatemala & 0,50 \\
\hline Honduras & 0,45 \\
\hline México & 0,68 \\
\hline Panamá & 0,61 \\
\hline Paraguay & 0,53 \\
\hline Perú & 0,65 \\
\hline República Dominicana & 0,57 \\
\hline Uruguay & 0,79 \\
\hline América Latina & 0,66 \\
\hline OCDE & 0,83 \\
\hline
\end{tabular}

Fuente: Análisis Telecom Advisory Services. 
La combinación de estos dos índices permite crear un índice compuesto de resiliencia del Estado para enfrentar la disrupción de la pandemia (Cuadro 12).

Cuadro 12. Índice de resiliencia del Estado (2018)

\begin{tabular}{|c|c|}
\hline País & Índice \\
\hline Argentina & 41,88 \\
\hline Bolivia & 25,97 \\
\hline Brasil & 41,64 \\
\hline Chile & 54,98 \\
\hline Colombia & 41,53 \\
\hline Ecuador & 39,13 \\
\hline El Salvador & 26,85 \\
\hline Guatemala & 21,16 \\
\hline Honduras & 18,83 \\
\hline México & 43,55 \\
\hline Panamá & 39,85 \\
\hline Paraguay & 30,47 \\
\hline Perú & 37,36 \\
\hline República Dominicana & 30,25 \\
\hline Uruguay & 47,07 \\
\hline América Latina & 40,36 \\
\hline OCDE & 66,17 \\
\hline
\end{tabular}

Fuente: Análisis Telecom Advisory Services. 
Como en el caso de resiliencia de digitalización de hogares, algunos Estados de la región parecen estar mejor posicionados para afrontar la disrupción: Chile, Uruguay, México, Brasil y Argentina.

\section{Conclusión}

El propósito de este artículo ha sido analizar la importancia de la digitalización como factor mitigante de la disrupción de la pandemia, focalizado en la situación de América Latina.

Como ya está ocurriendo a escala mundial, las redes latinoamericanas están siendo afectadas por el aumento exponencial del tráfico. En particular, se identifica una disminución de velocidad en las redes de banda ancha, lo que, en caso de perpetuarse, podría generar efectos económicos adversos. Asimismo, la migración masiva al teletrabajo está saturando la capacidad de enrutadores Wi-Fi en el hogar, por un aumento de trabajo en la nube y de la videoconferencia.

En este sentido, la brecha digital representa un obstáculo para sectores importantes de la población que dependerá del acceso a Internet para recibir información sanitaria, descargar contenidos educativos, o adquirir bienes de manera electrónica. Además, se percibe que el uso de Internet en gran parte de los hogares se limita a herramientas de comunicación y a redes sociales, lo cual acota la utilidad de la digitalización para hacer frente a la pandemia.

En cuanto a la resiliencia del aparato productivo, la región presenta falencias en lo que respecta a la asimilación de tecnología en procesos productivos, en particular en las cadenas de aprovisionamiento. Por otra parte, la COVID-19 implica una disrupción adicional en el mercado laboral ocasionada por la proporción de trabajadores que puede migrar al teletrabajo. Nuestro análisis para el caso de Chile indica que más del $56 \%$ de los trabajadores se encuentran en una situación precaria al no poder asistir al lugar de trabajo y al no poder hacer la transición al teletrabajo. Por tanto, es fundamental trabajar sobre la restructuración de procesos operacionales del sector productivo para permitir incrementar el porcentaje de la población que pueda trabajar remotamente, así como enfatizar la capacitación de los sectores sociales vulnerables.

La resiliencia del Estado frente a la pandemia está basada en su capacidad para seguir funcionando en términos de procesos administrativos, así como para continuar entregando servicios públicos. El cálculo de un índice compuesto de resiliencia del aparato del Estado indica que, debido al trabajo de años en el desarrollo de gobierno electrónico, ciertas naciones de la región parecen estar mejor posicionadas para afrontar la disrupción: Chile, Uruguay, México, Brasil y Argentina.

En definitiva, reconociendo que la digitalización puede jugar un papel fundamental en mitigar los efectos de la pandemia, es importante que gobiernos, sector privado y sociedad civil trabajen de forma conjunta y puedan identificar aquellas áreas de trabajo que permitan mejorar el desempeño de ciertos componentes del ecosistema digital. Entre otras acciones, 
se recomienda trabajar en los siguientes ejes:

- Acelerar el despliegue de mayor cantidad de radiobases para banda ancha móvil, eliminando cualquier requerimiento de permisos para el despliegue de antenas.

- Asignar a operadores móviles espectro adicional de manera temporaria.

- Requerir a los proveedores de servicios de video streaming la reducción en el volumen de tráfico que estos generan a partir de la disminución de estándares en la definición técnica de contenidos.

- Examinar la necesidad de aumentar la porción de espectro no licenciado en las bandas superiores de $5 \mathrm{GHz}$ y $6 \mathrm{GHz}$ para resolver los cuellos de botella en los enrutadores Wi-Fi.

- Promover innovación en el desarrollo de plataformas que permitan superar las falencias en las cadenas de aprovisionamiento. Por ejemplo, estimular el desarrollo de empresas tecnológicas para que suministren una relación más eficiente entre proveedores logísticos y servicios de transporte.

- Estimular el sector productivo para que innove en la restructuración de procesos para permitir incrementar el porcentaje de la población que pueda trabajar remotamente.

- Enfatizar la capacitación de los sectores sociales más vulnerables para poder enfrentar la desocupación.

- Y fundamentalmente, comenzar a trabajar inmediatamente en un plan de resiliencia digital para hacer frente a futuras pandemias.

\section{Referencias bibliográficas}

Briglauer, Wolfgang y Klaus Gugler. 2018. Go for Gigabit? First evidence on economic benefits of (Ultra-) Fast broadband technologies in Europe. Center for European Economic Research Discussion Paper № 18-020.

Calatayud, Agustina y Raúl Katz. 2019. Cadena de Suministro 4.0: Mejores prácticas internacionales y Hoja de Ruta para América Latina. Washington, DC: Banco Interamericano de Desarrollo.

Carew, Diana Gehlhaus, Nicholas Martin, Marjory S. Blumenthal, Philip Armour y Jesse Lastunen. 2018. The Potential Economic Value of Unlicensed Spectrum in the $5.9 \mathrm{GHz}$ Frequency Band: Insights for Future Spectrum Allocation Policy. Santa Monica, CA: RAND Corporation, 2018. https://www.rand.org/pubs/research_reports/RR2720.html.

Centro Regional de Estudos para o Desenvolvimento da Sociedade da Informação. 2017. Pesquisa sobre o uso das Tecnologías de Informação e Comunicação nas empresas brasileiras - TIC empresas. 
Federal Communications Commission. 2020. FCC Grants Temporary Spectrum Access to Support Connectivity on Tribal Reservation During Covid-19 Pandemic. Washington, DC.

Ford, George. 2018. Is faster better? Quantifying the relationship between broadband speed and economic growth. Telecommunications Policy, 42(9), 766-777.

Gobierno de Argentina - INDEC. 2012. Encuestas Nacional sobre Innovación y Conducta Tecnológica (2004-2011). Buenos Aires.

Gobierno de Chile - Ministerio de Economía, Fomento y Turismo; División de Política Comercial e Industrial. 2017. Encuesta Longitudinal de Empresas. Santiago.

Gobierno de Colombia - Ministerio TIC y Cámara de Comercio de Bogotá. 2017. Observatorio de la Economía Digital de Colombia. Bogotá.

Gobierno de Colombia - Departamento Nacional de Planeamiento. 2017. Documento Técnico, Misión de Logística y Comercio Exterior. Bogotá.

Gobierno de Ecuador - Instituto Nacional de Estadística y Censos. 2015. Empresas y TIC: Módulo de TIC de las Encuestas de Manufactura y Minería, Comercio Interno y servicios. Quito.

Gobierno de Perú - Instituto Nacional de Estadística e Informática. 2017. Encuesta Económica Anual Tecnología de Información y Comunicación en las empresas. Lima.

Gobierno de México - Secretaría de Comunicaciones y Transporte (SCT), Subsecretaría de Transporte. 2017. Estadística Básica del Autotransporte Federal 2017. México, DF.

Grimes, Arthur, Cleo Ren y Philip Stevens. 2011. The need for speed: impacts of internet connectivity on firm productivity. Journal of Productivity Analysis, 37(2), 187-201.

Hevia, Constantino y Andy Neumeyer. 2020. A conceptual framework for analyzing the economic impact of COVID-19 and its policy implications. UNDP Latin America and the Caribbean, UNDP LAC C19 PDS N.1.

Katz, Raúl y Fernando Callorda. 2019. Assessment of the economic value of 10G networks. New York: Telecom Advisory Services.

Katz, Raúl, Juan Jung y Fernando Callorda. 2020a. El ecosistema digital y la digitalización de la producción en América Latina y el Caribe: Medición e Impacto Económico. Informe elaborado para CEPAL, febrero.

Katz, Raúl, Juan Jung y Fernando Callorda. 2020b. El estado de la digitalización de América Latina frente a la pandemia del COVID-19. Caracas: Observatorio CAF del Ecosistema Digital, abril.

Katz, Raúl. 2020. El estado de la digitalización de América Latina frente a la pandemia del COVID-19. Presentación webinar de ASIET, abril 29. 
Kongaut, Chatchai y Erik Bohlin. 2017. Impact of broadband speed on economic outputs: An empirical study of OECD countries. Economics and Business Review, 3(2), 12-32.

Mack-Smith, David. 2006. Next Generation Broadband in Scotland. Edinburgh: SQW Limited.

Rohman, Ibrahim Kholilul y Erik Bohlin. 2012. Socio-economic effects of broadband speed. Ericsson 3/221 01-FGB 10100003.

Srinivasan, Sri. 2020. Cisco Webex: Supporting customers during this unprecedented time, marzo.

World Bank. 2019. Doing Business 2018. Washington, DC.

\section{Nota sobre la presente versión}

El presente estudio ha sido elaborado en el marco de las actividades del Observatorio CAF del Ecosistema Digital. Su propósito es contribuir a la reflexión sobre el impacto de la pandemia de la COVID-19 y plantear propuestas para mitigar sus efectos en América Latina y el Caribe.

\section{Biografía de los Autores}

Raúl Katz (MS, Tecnología y Políticas de Comunicación, PhD, Administración de Empresas y Ciencias Políticas, Massachusetts Institute of Technology) es director de Investigación de Estrategia Empresarial en el Columbia Institute for Tele-Information y presidente de Telecom Advisory Services, LLC.

Fernando Callorda (BA, MA, Ciencias Económicas, Universidad de San Andrés-Argentina) es director de Proyectos en Telecom Advisory Services, investigador en la Red Nacional de Universidades Públicas de Argentina y profesor de Economía Política en la Universidad de La Matanza.

Juan Jung (MA, PhD, Ciencias Económicas, Universidad de Barcelona) es consultor en Telecom Advisory Services y profesor en la Universidad Complutense de Madrid. 


\section{Notas}

Se define como ecosistema digital un nuevo contexto industrial y de impacto económico y social resultante de la adopción masiva de tecnologías digitales de información y comunicación. El estudio del ecosistema digital involucra tres dimensiones: nuevos modos de producción de información y contenidos, diferentes comportamientos sociales relativos al uso y consumo de bienes, y un impacto económico y social más importante que el de tecnologías de información y comunicación consideradas de manera aislada (Katz 2015, Katz y Callorda 2018).

Estos índices reflejan los valores del Índice CAF de Desarrollo del Ecosistema Digital.

Por ejemplo, en la tercera semana de marzo la velocidad de descarga de Internet en Estados Unidos disminuyó 4,9\%; en Nueva York el descenso fue de 24\%

$\uparrow 3$ (Fuente: Ookla/Speedtest). El descenso se produjo como consecuencia del aumento inusitado de tráfico (en la red de ATT, el tráfico en un mes aumentó 27\%, y en el caso de Verizon, 22\%).

La plataforma Ookla/Speedtest mide la velocidad y la latencia de Internet sobre la base de la compilación de tests realizados por usuarios desde sus dispositivos. Los valores dependen del número de observaciones, reflejando la preocupación de

$\uparrow 4$ usuarios respecto a las métricas de Internet, el número de test para la banda ancha fija en los países analizados se incrementó entre 50\% y 150\% en las semanas de marzo, mientras que en la banda ancha móvil el incremento fue de alrededor del $20 \%$.

Detalles en FCC (2020).

De acuerdo con Sandvine, Netflix, por ejemplo, consume 12,6\% del tráfico de descarga de Internet.

$\uparrow 7 \quad$ Fuente: Srinivasan (2020).

$\uparrow 8$ Fuente: ITU World Telecommunication/ICT Indicators database. 
$\uparrow 9$ No se desmerece, sin embargo, el uso de WhatsApp para la comunicación de emergencias sanitarias.

¡10 App Annie es una empresa que opera una plataforma de inteligencia de mercado enfocada en la adopción de apps.

†1 Fuente: Gobierno de México - Secretaría de Comunicaciones y Transporte, Subsecretaría de Transporte (2017).

$\uparrow 12$ Fuente: Registro Nacional de Despacho de Carga del Gobierno de Colombia.

\13 Fuente: Gobierno de Colombia - Departamento Nacional de Planeamiento (2017).

†14 Ejemplos: CargoX, Fretebras, Busca Cargas y Truckpad (Brasil); Humber, Circular y Avancargo (Argentina). 